\title{
Single and pair production of heavy leptons in $E_{6}$ model
}

\author{
A. T. Alan* A. T. Tasci $\dagger$ and N. Karagot \\ Abant Izzet Baysal University, Department of Physics, 14280 Bolu, Turkey
}

(Dated: November 20, 2018)

\begin{abstract}
We investigate the single and pair production of new heavy leptons via string inspired $E_{6}$ model at future linear colliders. Signal and corresponding backgrounds for these leptons are studied. We have found that single production of heavy leptons is more relevant than that of pair production, as expected. For a maximal mixing value of 0.1 , the upper mass limits of $2750 \mathrm{GeV}$ in the single case and $1250 \mathrm{GeV}$ in the pair production case are obtained at $\sqrt{s}=3 \mathrm{TeV}$ collider option.

PACS numbers: 12.60.-i, 13.66.De, 14.60.-z
\end{abstract}

*Electronic address: alan a@ibu.edu.tr

${ }^{\dagger}$ Electronic address: tasci a@ibu.edu.tr

${ }^{\ddagger}$ Electronic address: karagoz n@ibu.edu.tr 


\section{INTRODUCTION}

The Standard Model (SM) describes many phenomena up to the energies that can be reached today. However, some problems like mass hierarchy and the number of fermion generations can not be addressed by the SM. Many models, which include new fermions and interactions, have been developed to deal with the mentioned shortcomings of the SM. The string inspired $E_{6}$ model is a well motivated one which includes extra gauge bosons and new fermions assigned to the 27-dimensional representation [1]. In the search for extensions of the SM the new heavy leptons play an important role. Many analysis have been done for the production of heavy leptons at future $e^{-} e^{+}[2,3,44,5]$, at hadron [6, 7, 8] and also at $e p$ colliders [9, 10]. The experimental upper bounds for the heavy lepton masses were found to be $44 \mathrm{GeV}$ by OPAL [11], $46 \mathrm{GeV}$ by ALEPH [12] and $90 \mathrm{GeV}$ by H1 [13] Collaborations. This means that, heavy leptons could be detected at future high energy colliders.

We have previously considered the single production of new heavy leptons via string inspired $E_{6}$ model in ep collisions [10]. In this study, we consider both the single and the pair production of heavy leptons separately by using the same model and taking into account the signal and background events at future linear colliders. The main parameters of these collider options were taken from Refs. [14, 15] and displayed in Table [.

\section{SINGLE PRODUCTION OF HEAVY LEPTONS}

The single production of heavy leptons $L$, in $e^{-} e^{+}$collisions occur through the $s$ and $t$ channel processes $e^{-} e^{+} \rightarrow L e^{+}$caused by the flavor changing neutral current (FCNC) Lagrangian:

$$
\mathcal{L}_{\mathrm{nc}}=g_{z} \sin \theta_{\text {mix }} \psi_{L} \gamma^{\mu}\left(1+\gamma_{5}\right) \psi_{e} Z^{\mu}+\text { h.c. }
$$

where $\sin \theta_{\text {mix }}$ are the mixing angles between right handed components of the ordinary and new heavy charged leptons. The order of the mixings of the ordinary and heavy leptons are known to be $\sin ^{2} \theta_{\text {mix }} \approx 10^{-2}-10^{-3}$, which comes from low energy phenomenological calculations and the high precision measurements of the $Z$ properties at linear colliders 16, 17, 18]. We use the parameter $b_{l L Z}$ to denote the mixing angles and take 0.1 as an upper value in the numerical calculations. 
The differential production cross section takes the form,

$$
\begin{aligned}
\frac{d \sigma}{d t}= & \frac{\pi \alpha^{2} b_{l L Z}^{2}}{\sin ^{4} \theta_{w} \cos ^{4} \theta_{w} s^{2}}\left[\frac{4\left(m^{2}-s-t\right)(s+t)\left(s-M_{Z}^{2}\right)\left(t-M_{Z}^{2}\right)\left(a_{e}-v_{e}\right)^{2}}{\left[\left(s-M_{Z}^{2}\right)^{2}+M_{Z}^{2} \Gamma_{Z}^{2}\right]\left[\left(t-M_{Z}^{2}\right)^{2}+M_{Z}^{2} \Gamma_{Z}^{2}\right]}\right. \\
& -\frac{\left(m^{2}-s-t\right)(s+t)\left(a_{e}-v_{e}\right)^{2}+t\left(m^{2}-t\right)\left(a_{e}+v_{e}\right)^{2}}{\left(s-M_{Z}^{2}\right)^{2}+M_{Z}^{2} \Gamma_{Z}^{2}} \\
& \left.+\frac{\left[\left[(s+t)^{2}+s^{2}-(2 s+t) m^{2}\right]\left(a_{e}^{2}+v_{e}^{2}\right)+2 t\left(m^{2}-2 s-t\right) a_{e} v_{e}\right]}{\left(t-M_{Z}^{2}\right)^{2}+M_{Z}^{2} \Gamma_{Z}^{2}}\right]
\end{aligned}
$$

where $a_{e}=-\frac{1}{2}$ and $v_{e}=-\frac{1}{2}+2 \sin ^{2} \theta_{w}, \Gamma_{Z}$ is the decay width and $M_{Z}$ is the mass of $Z$ boson, $s$ and $t$ are Mandelstam variables.

In Fig. 1, we display the total cross sections as function of the heavy lepton masses, for the three center of mass energies of the proposed options. After their production, the heavy leptons will decay via the neutral current process $L \rightarrow l Z$, where $l=e, \mu, \tau$. The branching ratios for these processes would be around $33 \%$ for each channel.

In Tables II III and IV we presented the single production cross sections $\left(\sigma \times \mathrm{BR}_{1}\right)$, signal and background cross sections depending on the heavy lepton mass $m_{L}$, for $0.5,1$ and $3 \mathrm{TeV}$ energy $e^{-} e^{+}$colliders, respectively. The branching ratios $\mathrm{BR}_{1}$ and $\mathrm{BR}_{2}$ refer to $\operatorname{BR}(L \rightarrow Z e)$ and $\operatorname{BR}\left(Z \rightarrow e^{+} e^{-}, \mu^{+} \mu^{-}\right)$. The significance of signal and background is defined as $S / \sqrt{S+B}$, here $S$ and $B$ are the signal and background number of events. The total decay widths of the heavy leptons are given in the last column of the tables. As seen from these tables, the $S / \sqrt{S+B}$ values are higher than five, which is enough for observability, up to the center of mass energies of the colliders. Single production of heavy lepton is feasible up to the center of mass energies of the $e^{-} e^{+}$colliders even with smaller mixing coupling values. For example, if we take at least 10 signal events and $S / \sqrt{S+B} \geq 5$ as discovery criteria, the ILC with $\sqrt{s}=0.5 \mathrm{TeV}$ can probe mixing values of $b_{l L Z}=0.032$ for $350 \mathrm{GeV}$ leptons. The same couplings can be probed at $\sqrt{s}=1$ and $\sqrt{s}=3 \mathrm{TeV}$ for even greater masses such as 800 and $2750 \mathrm{GeV}$.

We applied a cut of $\left|m_{Z e}-m_{L}\right|<10 \mathrm{GeV}$ in order to form the signal and reduce the background for the SM background process $e^{-} e^{+} \rightarrow e^{-} Z e^{+}$. Fig. 2] shows the $p_{T}$ distributions at three different linear colliders. In Figs. 3, 4and 5, we give the invariant mass distributions $m_{Z e}$ with cut $p_{T}^{e^{-}, j}>10 \mathrm{GeV}$ at $\sqrt{s}=0.5,1$ and $3 \mathrm{TeV}$, respectively. Figs. [3 and 4 have an increasing character for a cut $10 \mathrm{GeV}$ interestingly, but lose this character for higher cuts. For instance, all of the three distributions in Figs. 3. 5 have decreasing characters with Jacobian peaks around 150-200 GeV for a cut value of $80 \mathrm{GeV}$. 


\section{PAIR PRODUCTION OF HEAVY LEPTONS}

Pair production of heavy leptons in $E_{6}$ occur through the $t$-channel flavor changing neutral current process $e^{-} e^{+} \rightarrow L^{-} L^{+}$, and the differential cross section for this process is given by,

$$
\frac{d \sigma}{d t}=\frac{4 \pi \alpha^{2} b_{l L Z}^{4}}{s^{2} M_{Z}^{4}\left[\left(t-M_{Z}^{2}\right)^{2}+M_{Z}^{2} \Gamma_{Z}^{2}\right]}\left[\left(m^{2}-t\right)^{2} m^{4}+4 M_{Z}^{2} m^{4} s+4\left(s+t-m^{2}\right)^{2} M_{Z}^{4}\right] .
$$

The total cross sections as functions of heavy lepton masses $m_{L}$, are displayed in Fig. 6. Signal and background cross sections depending again on the heavy lepton masses, are presented in Tables $\mathbf{V}$ VI and VII at 0.5, 1 and $3 \mathrm{TeV}$, respectively. For the pair production of heavy leptons at linear colliders we expect of order of $10^{2}-10^{3}$ signal events for 1250 $\mathrm{GeV}$ leptons for the coupling value of $b_{l L Z}=0.1$. On the other hand, the lower limit of the coupling which can be probed by pair production at linear colliders is found to be 0.05 .

We applied an initial cut on the electron and jet transverse momentum $p_{T}^{e, j}>20 \mathrm{GeV}$ for the signal and background analysis. Fig. 7 shows the $p_{T}$ distributions of the background at the colliders. The distribution of invariant mass $m_{Z e^{-}}$is presented in Figs. 8] 9] and 10] at $\sqrt{s}=0.5,1$ and 3 , respectively.

We have used the high energy package CompHEP for calculations of background cross sections reported in this study [19].

\section{CONCLUSIONS}

This study proves that linear colliders can test the existence of single and pair production of heavy leptons. The production of a single heavy lepton is more relevant than the pair production. Namely, in the case of $\sqrt{s}=3 \mathrm{TeV}$ option, we expect 257 single events for $b_{l L Z}=0.1$ and 26 single events for $b_{l L Z}=0.032$ for $2750 \mathrm{GeV}$ (which is the upper bound) leptons. In the case of pair production, for $b_{l L Z}=0.1$ we expect $10^{2}-10^{3}$ events for 1250 $\mathrm{GeV}$ (upper value) leptons, while no pair event can be observed for $b_{l L Z}=0.032$, since the pair production cross section is suppressed by the fourth power of mixing couplings. 


\section{Acknowledgments}

This study was partially supported by Abant Izzet Baysal University Research fund.

[1] J. L. Hewett and T. G. Rizzo, Phys. Rept. 183, 193 (1989).

[2] F. M. L. Almeida, J. H. Lopes, J. A. Martins Simoes and C. M. Porto, Phys. Rev. D 44, 2836 (1991).

[3] F. M. L. Almeida, J. H. Lopes, J. A. Martins Simoes, P. P. Queiroz Filho and A. J. Ramalho, Phys. Rev. D 51, 5990 (1995)

[4] F. M. L. Almeida, Y. A. Coutinho, J. A. Martins Simoes and M. A. B. do Vale, Phys. Rev. D 63, $075005(2001)$

[5] F. M. L. Almeida, Y. A. Coutinho, J. A. Martins Simoes, S. Wulck and M. A. B. do Vale, Eur. Phys. J. C 30, 327 (2003)

[6] P. H. Frampton, D. Ng, M. Sher and Y. Yuan, Phys. Rev. D 48, 3128 (1993)

[7] Y. A. Coutinho, J. A. Martins Simoes, C. M. Porto and P. P. Queiroz Filho, Phys. Rev. D $\mathbf{5 7 ,} 6975$ (1998).

[8] J. E. Cieza Montalvo and P. P. de Queiroz Filho, Phys. Rev. D 66, 055003 (2002).

[9] T. G. Rizzo, Phys. Lett. B 188, 95 (1987).

[10] A. T. Alan, A. T. Tasci and O. Cakir, Acta Phys. Polon. B 35, 2199 (2004).

[11] M. Z. Akrawy et al. [OPAL Collaboration], Phys. Lett. B 240, 250 (1990).

[12] D. Decamp et al. [ALEPH Collaboration], Phys. Rept. 216, 253 (1992).

[13] T. Ahmed et al. [H1 Collaboration], Phys. Lett. B 340, 205 (1994).

[14] E. Accomando et al. [CLIC Physics Working Group], arXiv:hep-ph/0412251.

[15] International Linear Collider-Technical Review Report, ILC-TRC/2003 Report (2003), http://www.slac.stanford.edu/xorg/ilc-trc/2002/index.html

[16] P. Bamert, C. P. Burgess and I. Maksymyk, Phys. Lett. B 356, 282 (1995)

[17] F. M. L. Almeida, Y. A. Coutinho, J. A. Martins Simoes and M. A. B. do Vale, Phys. Rev. D 62, $075004(2000)$

[18] E. Nardi, E. Roulet and D. Tommasini, Phys. Lett. B 327, 319 (1994)

[19] A. Pukhov et al., "CompHEP: A package for evaluation of Feynman diagrams and integration 
over multi-particle phase space.", arXiv:hep-ph/9908288. 
TABLE I: The main parameters of the future $e^{-} e^{+}$colliders.

\begin{tabular}{ccc}
\hline \hline$e^{-} e^{+}$colliders & $\sqrt{s}(\mathrm{TeV})$ & $\mathcal{L}\left(\mathrm{cm}^{-2} \mathrm{~s}^{-1}\right)$ \\
\hline ILC & 0.5 & $10^{34}-10^{35}$ \\
CLIC & 1.0 & $10^{34}-10^{35}$ \\
CLIC & 3.0 & $10^{34}-10^{35}$ \\
\hline \hline
\end{tabular}

TABLE II: The signal and background cross sections and $S / \sqrt{S+B}$ depending on the heavy lepton masses with $\sqrt{s}=0.5 \mathrm{TeV}$.

\begin{tabular}{ccccccc}
\hline \hline$m_{L}(\mathrm{GeV})$ & $\sigma(\mathrm{pb})$ & $\sigma \times \mathrm{BR}_{1}(\mathrm{pb})$ & $\sigma \times \mathrm{BR}_{1} \times \mathrm{BR}_{2}(\mathrm{pb})$ & $\sigma_{B} \times 10^{-3}(\mathrm{pb})$ & $S / \sqrt{S+B}$ & $\Gamma_{\text {Total }}(\mathrm{GeV})$ \\
\hline 100 & 1.49 & 0.49 & 0.016 & 0.63 & 39 & 0.008 \\
200 & 1.29 & 0.42 & 0.014 & 1.25 & 36 & 0.589 \\
300 & 0.96 & 0.32 & 0.010 & 1.34 & 30 & 2.159 \\
400 & 0.50 & 0.17 & 0.005 & 1.76 & 20 & 5.183 \\
\hline \hline
\end{tabular}

TABLE III: The signal and background cross sections and $S / \sqrt{S+B}$ depending on the heavy lepton masses with $\sqrt{s}=1 \mathrm{TeV}$.

\begin{tabular}{ccccccc}
\hline \hline$m_{L}(\mathrm{GeV})$ & $\sigma(\mathrm{pb})$ & $\sigma \times \mathrm{BR}_{1}(\mathrm{pb})$ & $\sigma \times \mathrm{BR}_{1} \times \mathrm{BR}_{2}(\mathrm{pb})$ & $\sigma_{B} \times 10^{-4}(\mathrm{pb})$ & $S / \sqrt{S+B}$ & $\Gamma_{\text {Total }}(\mathrm{GeV})$ \\
\hline 100 & 1.50 & 0.49 & 0.016 & 0.99 & 40 & 0.008 \\
300 & 1.38 & 0.45 & 0.015 & 2.89 & 38 & 2.159 \\
500 & 1.13 & 0.37 & 0.012 & 2.83 & 35 & 10.146 \\
700 & 0.76 & 0.25 & 0.008 & 3.33 & 28 & 27.834 \\
900 & 0.27 & 0.09 & 0.003 & 5.39 & 16 & 59.108 \\
\hline \hline
\end{tabular}


TABLE IV: The signal and background cross sections and $S / \sqrt{S+B}$ depending on the heavy lepton masses with $\sqrt{s}=3 \mathrm{TeV}$.

\begin{tabular}{ccccccc}
\hline \hline$m_{L}(\mathrm{GeV})$ & $\sigma(\mathrm{pb})$ & $\sigma \times \mathrm{BR}_{1}(\mathrm{pb})$ & $\sigma \times \mathrm{BR}_{1} \times \mathrm{BR}_{2}(\mathrm{pb})$ & $\sigma_{B} \times 10^{-5}(\mathrm{pb})$ & $S / \sqrt{S+B}$ & $\Gamma_{\text {Total }}(\mathrm{GeV})$ \\
\hline 250 & 1.49 & 0.49 & 0.016 & 21.06 & 40 & 1.22 \\
750 & 1.40 & 0.46 & 0.015 & 3.97 & 39 & 34.23 \\
1250 & 1.23 & 0.41 & 0.013 & 3.32 & 37 & 158.19 \\
1750 & 0.98 & 0.32 & 0.011 & 4.50 & 33 & 433.67 \\
2250 & 0.65 & 0.22 & 0.007 & 6.35 & 27 & 921.23 \\
2750 & 0.24 & 0.08 & 0.003 & 10.05 & 16 & 1681.41 \\
\hline \hline
\end{tabular}

TABLE V: The signal and background cross sections and $S / \sqrt{S+B}$ depending on the heavy lepton masses with $\sqrt{s}=0.5 \mathrm{TeV}$.

\begin{tabular}{|c|c|c|c|c|c|c|}
\hline$m_{L}(\mathrm{GeV})$ & $\sigma(\mathrm{pb})$ & $\sigma \times \mathrm{BR}_{1}(\mathrm{pb})$ & $\sigma \times \mathrm{BR}_{1} \times \mathrm{BR}_{2}(\mathrm{pb})$ & $\sigma_{B} \times 10^{-8}(\mathrm{pb})$ & $S / \sqrt{S+B}$ & $\Gamma_{\text {Total }}(\mathrm{GeV})$ \\
\hline 100 & 0.36 & 0.12 & 0.0039 & 0.005 & 20 & 0.01 \\
\hline 150 & 0.35 & 0.12 & 0.0038 & 2.49 & 20 & 0.13 \\
\hline 200 & 0.37 & 0.12 & 0.0040 & 5.03 & 20 & 0.59 \\
\hline 240 & 0.27 & 0.09 & 0.0029 & 1.25 & 17 & 1.22 \\
\hline
\end{tabular}

TABLE VI: The signal and background cross sections and $S / \sqrt{S+B}$ depending on the heavy lepton masses with $\sqrt{s}=1 \mathrm{TeV}$.

\begin{tabular}{|c|c|c|c|c|c|c|}
\hline$m_{L}(\mathrm{GeV})$ & $\sigma(\mathrm{pb})$ & $\sigma \times \mathrm{BR}_{1}(\mathrm{pb})$ & $\sigma \times \mathrm{BR}_{1} \times \mathrm{BR}_{2}(\mathrm{pb})$ & $\sigma_{B} \times 10^{-7}(\mathrm{pb})$ & $S / \sqrt{S+B}$ & $\Gamma_{\text {Total }}(\mathrm{GeV})$ \\
\hline 100 & 0.43 & 0.14 & 0.0047 & 0.004 & 22 & 0.01 \\
\hline 200 & 0.44 & 0.15 & 0.0048 & 1.51 & 22 & 0.59 \\
\hline 300 & 0.56 & 0.18 & 0.0061 & 3.57 & 25 & 2.16 \\
\hline 400 & 0.94 & 0.31 & 0.0102 & 5.12 & 32 & 5.18 \\
\hline
\end{tabular}


TABLE VII: The signal and background cross sections and $S / \sqrt{S+B}$ depending on the heavy lepton masses with $\sqrt{s}=3 \mathrm{TeV}$.

\begin{tabular}{|c|c|c|c|c|c|c|}
\hline$m_{L}(\mathrm{GeV})$ & $\sigma(\mathrm{pb})$ & $\sigma \times \mathrm{BR}_{1}(\mathrm{pb})$ & $\sigma \times \mathrm{BR}_{1} \times \mathrm{BR}_{2}(\mathrm{pb})$ & $\sigma_{B} \times 10^{-6}(\mathrm{pb})$ & $S / \sqrt{S+B}$ & $\Gamma_{\text {Total }}(\mathrm{GeV})$ \\
\hline 250 & 0.47 & 0.16 & 0.0051 & 0.90 & 23 & 1 \\
\hline 500 & 0.61 & 0.20 & 0.0066 & 4.04 & 26 & 10 \\
\hline 750 & 1.51 & 0.50 & 0.0164 & 3.92 & 41 & 34 \\
\hline 1000 & 3.96 & 1.31 & 0.0431 & 1.18 & 66 & 81 \\
\hline 1250 & 7.79 & 2.57 & 0.0848 & 16.20 & 92 & 158 \\
\hline
\end{tabular}

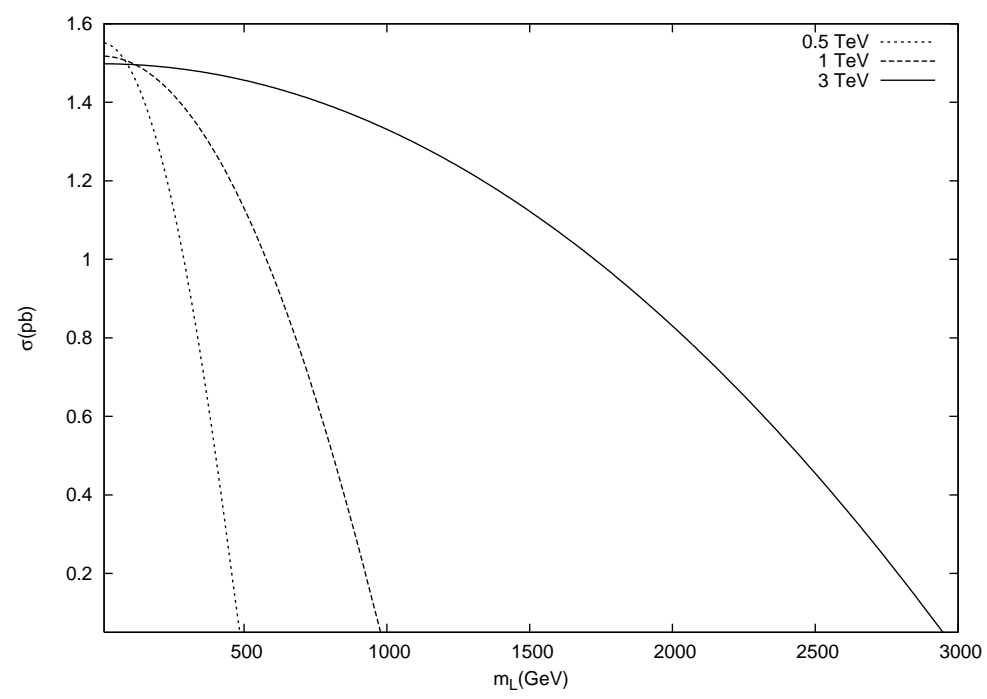

FIG. 1: The total cross sections as function of the heavy lepton masses, for the single production of heavy lepton with $\sqrt{s}=0.5 \mathrm{TeV}, 1 \mathrm{TeV}$ and $3 \mathrm{TeV}$. 


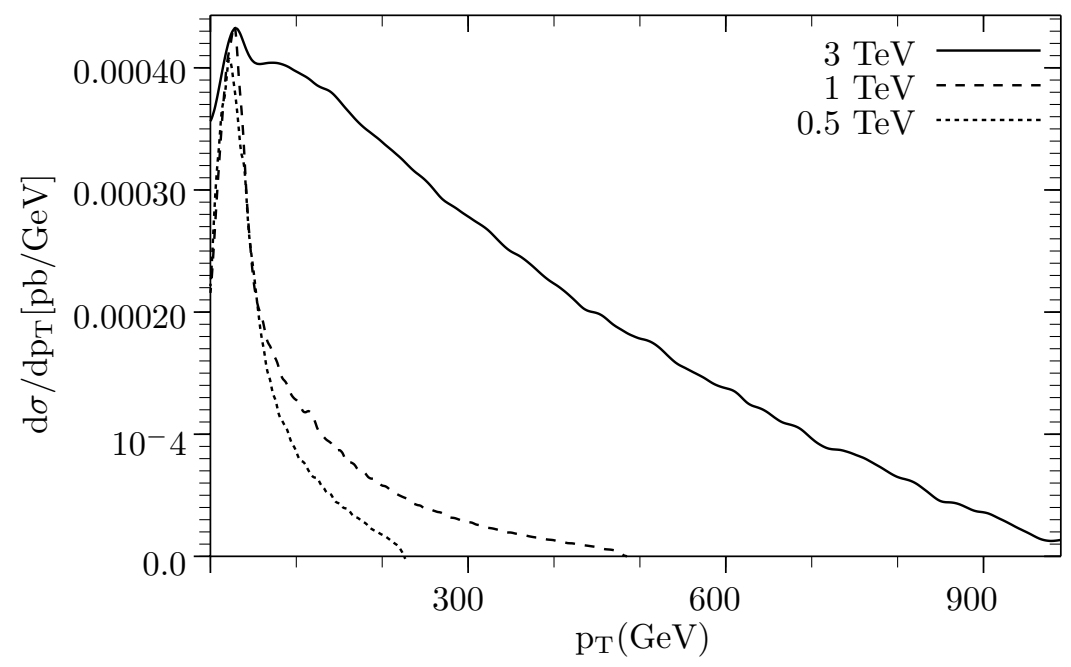

FIG. 2: $p_{T}$ distribution of the background at $\sqrt{s}=0.5 \mathrm{TeV}, 1 \mathrm{TeV}$ and $3 \mathrm{TeV}$.

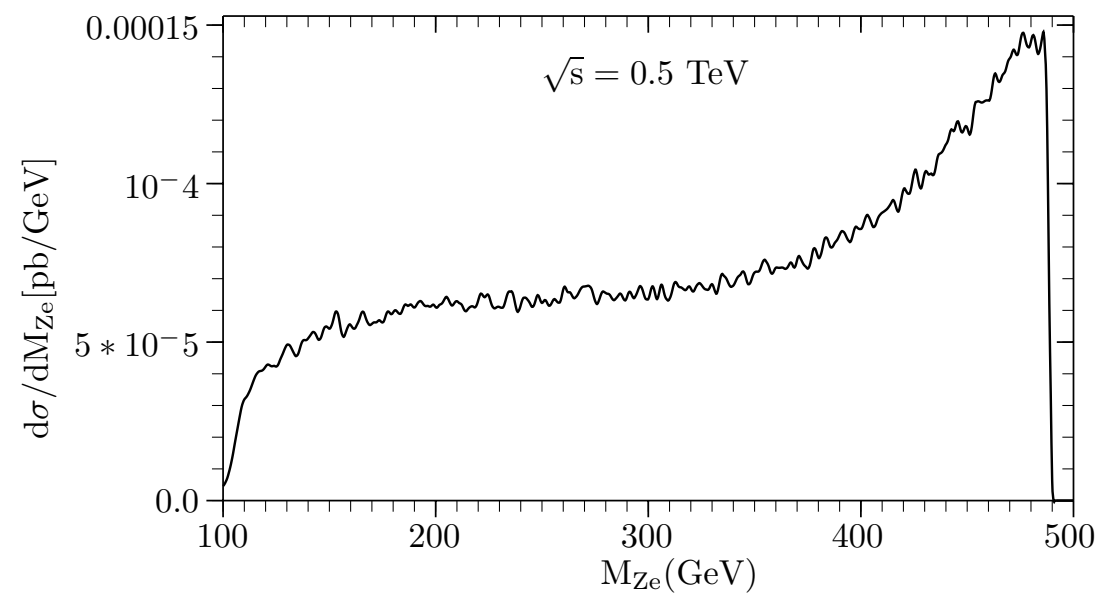

FIG. 3: The invariant mass distribution of the $Z e^{-}$system for the background at $\sqrt{s}=0.5 \mathrm{TeV}$. 


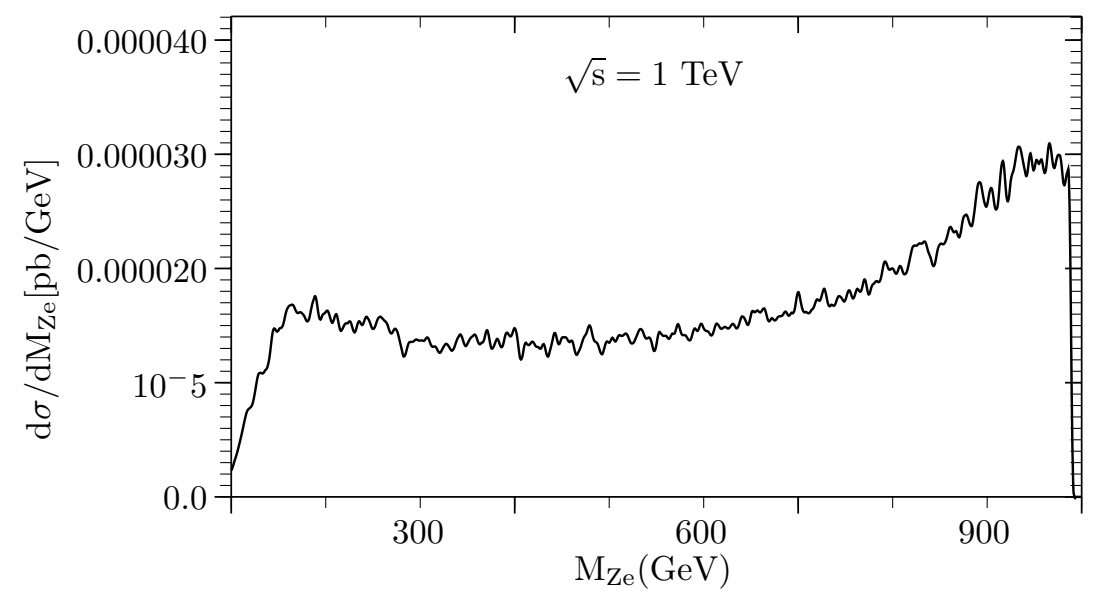

FIG. 4: The invariant mass distribution of the $Z e^{-}$system for the background at $\sqrt{s}=1 \mathrm{TeV}$.

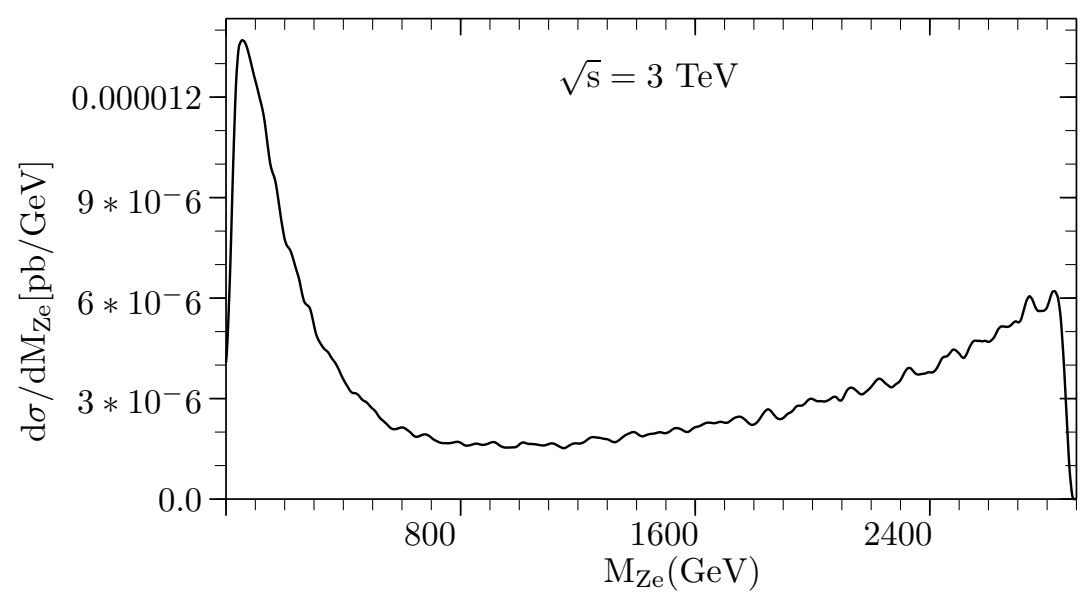

FIG. 5: The invariant mass distribution of the $Z e^{-}$system for the background at $\sqrt{s}=3 \mathrm{TeV}$. 


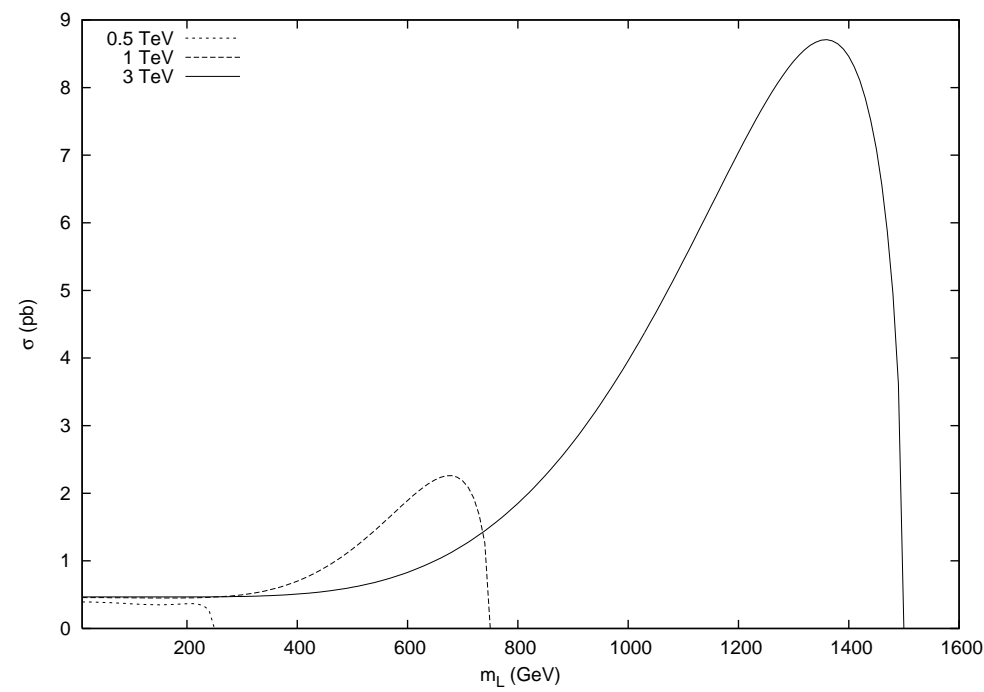

FIG. 6: The total cross sections as function of the heavy lepton masses, for the pair production of heavy lepton with $\sqrt{s}=0.5 \mathrm{TeV}, 1 \mathrm{TeV}$ and $3 \mathrm{TeV}$.

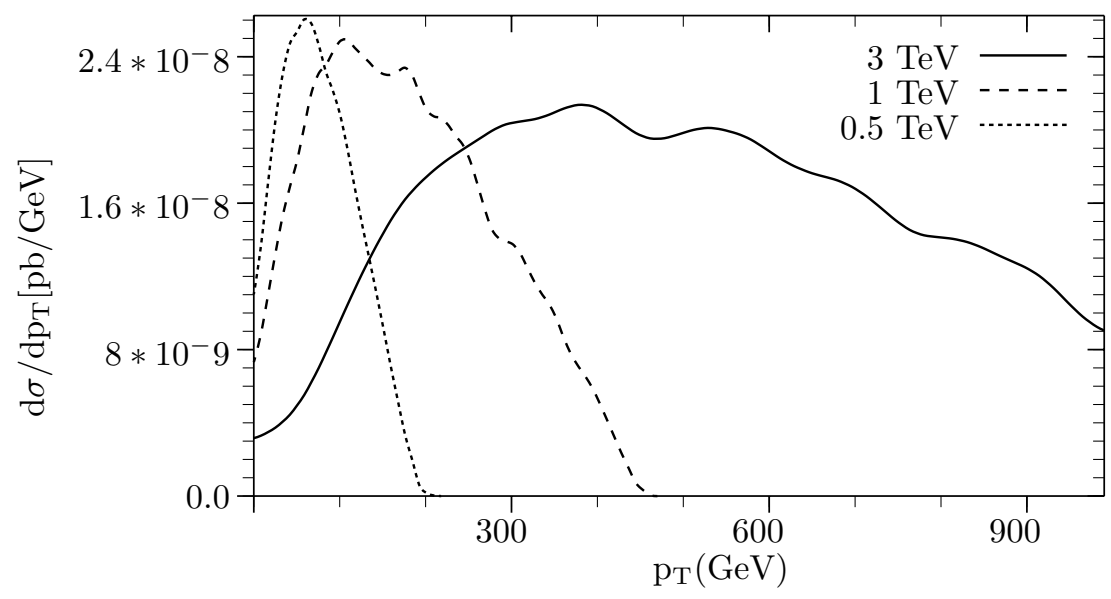

FIG. 7: $p_{T}$ distribution of the background at $\sqrt{s}=0.5 \mathrm{TeV}, 1 \mathrm{TeV}$ and $3 \mathrm{TeV}$. 


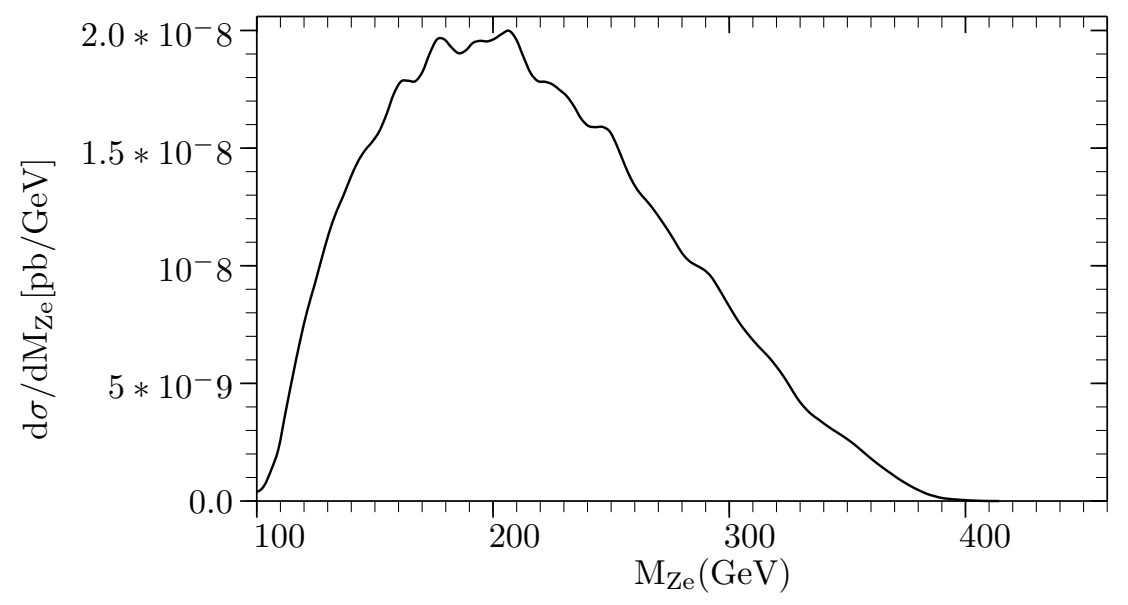

FIG. 8: The invariant mass distribution of the $Z e^{-}$system for the background at $\sqrt{s}=0.5 \mathrm{TeV}$.

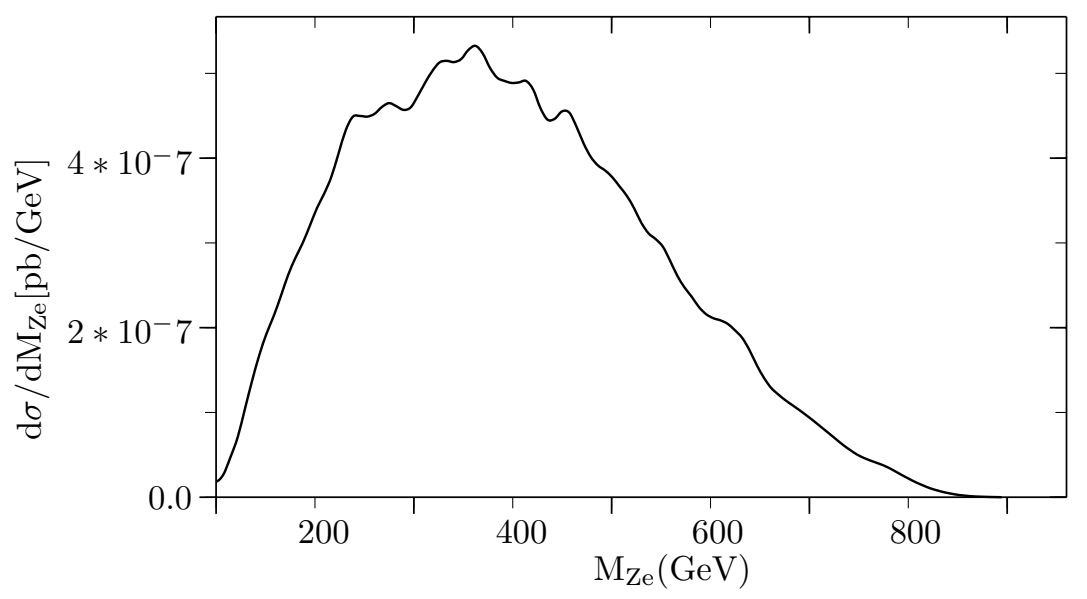

FIG. 9: The invariant mass distribution of the $Z e^{-}$system for the background at $\sqrt{s}=1 \mathrm{TeV}$. 


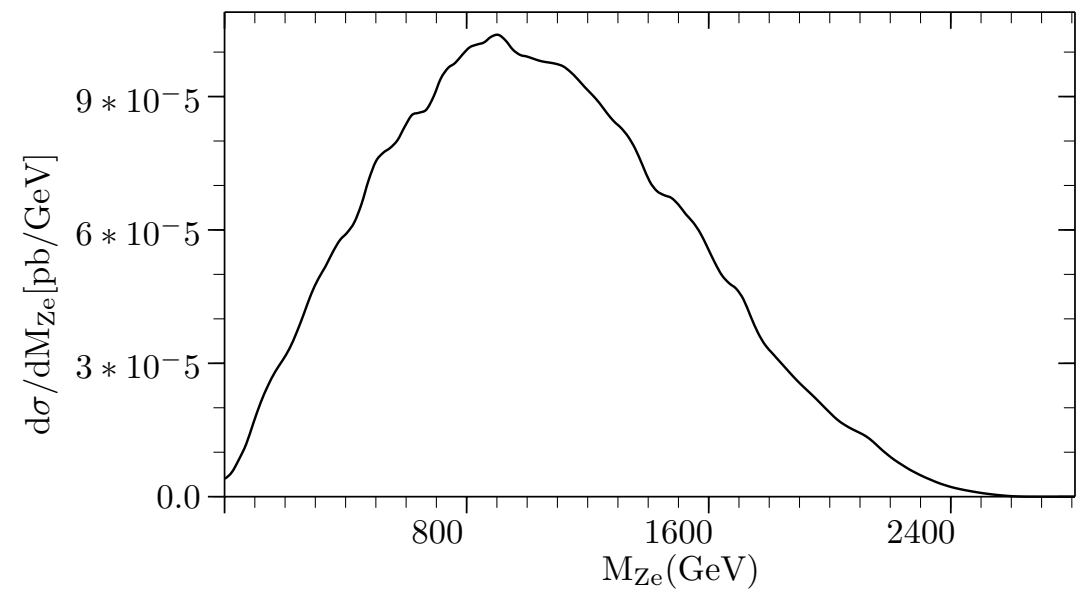

FIG. 10: The invariant mass distribution of the $Z e^{-}$system for the background at $\sqrt{s}=3 \mathrm{TeV}$. 